Objectives: to evaluate (i) whether quantitative and qualitative differences in the activation of the IFN signature can be found in RA patients depending on the clinical stage and (ii) if these differences may be linked to a clinical relevance of the IFN signature.

Methods: expression of IFI44, IFI44L, IFI6 and MX1 was determined in peripheral blood in 98 RA patients and 28 controls. RA patients were classified into groups according to their clinical stage and treatments received: very early RA (VERA, recruited at onset and not exposed to any treatment), bDMARD-naïve (patients on csDMARD treatment) and bDMARD (patients on biological treatment, all antiTNF $\alpha$ agents). An additional group of 13 RA patients candidate for TNF $\alpha$-blockade was also recruited and samples were taken before and after anti-TNF $\alpha$ treatment. The associations among IRGs were evaluated by network and principal component analyses.

Results: all IRGs was increased in RA, although differences were noted among them. The IFN score was increased in all RA groups (VERA, bDMARD-naïve and bDMARD), but differences in their degree of activation and in the relationships among IRGs were observed. VERA patients exhibited a lower activation of the IFN signature and a distinct picture of the structure of the IRG network (figure 1) compared to both their established disease-counterparts and the HC group. The IFN score correlated the accumulated DAS28 over one year $(r=0.593, p=0.025)$ and it was found to be a predictor of a good clinical outcome (EULAR good clinical response) in VERA ( $A \cup C=0.917, p=0.004$ ). However, no differences in the IFN score were observed between the bDMARD-naïve and bDMARD groups, but opposite associations with the clinical parameters were noted. Interestingly, the correlations among IRGs delineate different pictures between these two groups. The IFN score at baseline predicted poor clinical outcome upon TNF $\alpha$-blockade. Although no absolute changes in the IFN score were found, TNF $\alpha$-blockade shifted the associations among IRGs. These differences mirrored those found when comparing bDMARD-naïve and bDMARD groups.
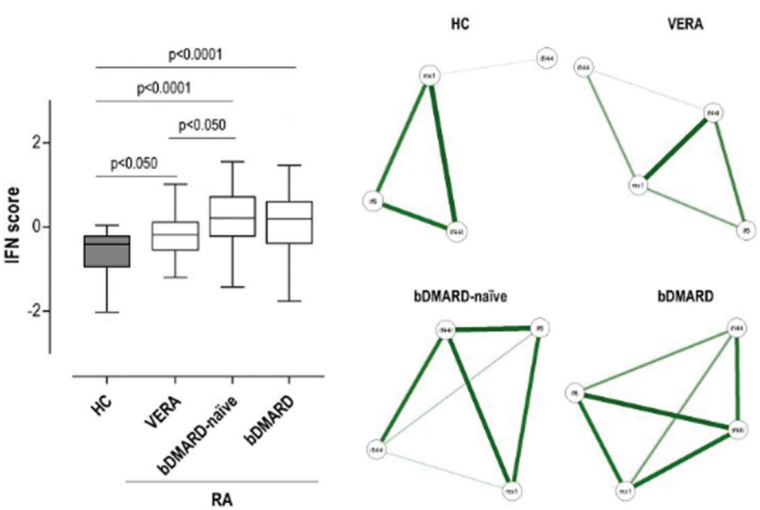

Abstract AB0041 - Figure 1

Conclusions: a certain heterogeneity within the IFN signature can be recognised in RA, depending on the clinical stage. The structure of the IFN signature may be a potential explanation for the controversy in this field and may represent a limitation for its use as a clinical biomarker.

Disclosure of Interest: None declared

DOI: 10.1136/annrheumdis-2018-eular.3195

\section{AB0042 ESSENTIAL OIL EXTRACTED FROM CHAMAECYPARIS OBTUSA ATTENUATES THE SEVERITY OF COLLAGEN- INDUCED ARTHRITIS}

J. Lee ${ }^{1}$, Y. Park ${ }^{2}$, C.-H. Yoon ${ }^{2} .{ }^{1}$ Division of Rheumatology, Department of Internal Medicine, College of Medicine, The Catholic University of Korea, Uijeongbu Saint Mary's Hospital; ${ }^{2}$ Division of Rheumatology, Department of Internal Medicine, College of Medicine, The Catholic University of Korea, Seoul, Korea., Seoul, Korea, Republic of Ireland

Background : Chamaecyparis obtusa (C. obtusa) is a tropical tree species found in Japan and the southern region of South Korea. Although the biological activities of essential oil extracted from C. obtusa (EOCO) are not yet fully understood, essential oil has been shown to have antibacterial activities and anti-inflammatory effects.
Objectives: We investigated the effect of essential oil extracted from Chamaecyparis obtusa on the development of collagen-induced arthritis $(\mathrm{CIA})$, a well-characterised preclinical model of human RA.

Methods: DBA1/J mice were injected intraperitoneally with EOCO dissolved in dimethyl sulfoxide (DMSO) 3 times a week for 5 weeks after type II collagen (CII) immunisation. Arthritis severity was evaluated by clinical and histological parameters. Serum anti-CII antibody levels were assayed by ELISA. linterleukin-17 (IL17) expressions in the joints were determined by immunohistochemistry. Effect of EOCO on Th17 cells and natural killer (NK) cells was analysed using flow cytometry.

Results: EOCO markedly reduced the clinical and histological severity of CIA Serum anti-CII antibody levels and the expression of IL-17 in the joints were significantly lower in mice treated with EOCO than those treated with DMSO alone. EOCO inhibited the differentiation of Th17 cells in total splenocytes, not in isolated $\mathrm{CD}^{+} \mathrm{T}$ cells, from mice. EOCO increased the production of granzyme $\mathrm{B}$ and perforin in NK cells.

Conclusions: Our data demonstrate a protective effect of EOCO on the development of CIA through the inhibition of Th17 cell differentiation. This effect may be related with NK cell activity enhanced by EOCO.

\section{REFERENCES:}

[1] Hong EJ, Na KJ, Choi IG, Choi KC, Jeung EB. Antibacterial and antifungal effects of essential oils from coniferous trees. Biol Pharm Bul 2004;27:863-6.

[2] An BS, Kang JH, Yang H, Jung EM, Kang HS, Choi IG, et al. Anti-inflammatory effects of essentia oils from Chamaecyparis obtusa via the cyclooxygenase-2 pathway in rats. Mol Med Rep 2013;8:255-9.

Acknowledgements: This study was carried out with the support of 'Forest Science and Technology Projects (Project No. S111114L020100)' provided by Korea Forest Service.

Disclosure of Interest: None declared

DOI: 10.1136/annrheumdis-2018-eular.1413

\section{AB0043 1 THE ELASTICITY PROPERTIES OF PROBIOTIC BACTERIA WALL ASSOCIATED WITH BENEFICIAL MODULATORY ACTIVITY ON INNATE IMMUNITY OF THE HOST}

L.P. Babenko, R.V. Bubnov, L.M. Lazarenko, M.Y. Spivak. Interferon, Zabolotny Institute of Microbiology and Virology, National Academy of Sciences of Ukraine, Kyiv, Ukraine

Background: Probiotics have tremendous potential to develop healthy diets and integrated approach for immunity-related diseases treatment and prevention; $;^{1,2}$ are effective actors in distant sites $^{3}$ with strong potential for applications in rheumatology. The cell wall of probiotic bacteria plays an essential role in many aspects of modulating beneficial immune response; ${ }^{4}$ its elasticity properties associated with probiotic beneficial effects and can warrant to stratify strains on their modulatory activity on innate immunity to justify individualised and personalised approach for nutrition and prevention.

Objectives: The aim was to study the effect of lactic acid bacteria (LAB) and bifidobacteria strains on phagocytic system cells functional activity and immunoregu latory cytokines synthesis in vitro in regards to the bacteria surface properties as cell walls elasticity using atomic force microscopy (AFM).

Methods: We conducted experimental studies on BALB/c line mice 18-20 g weight using lyophilized strains of LAB - Lactobacillus acidophilus IMV B-7279, L. casei IMV B-7280, L. delbrueckii subsp. bulgaricus IMV B-7281 and bifidobacteria - Bifidobacterium animalis VKL, B. animalis VKB. We cultivated the macrophages received from the peritoneal cavity of mice by common method individually with the strains of $L A B$ and bifidobacteria. We estimated the impact of $L A B$ and bifidobacteria strains on the functional activity of peritoneal cavity macrophages using the conventional methods of study oxygen-dependent bactericidal activity, nitric oxide production, their effect on the immunoregulatory cytokines. We used AFM scanning to estimate bacteria cell walls elasticity.

Results: All strains demonstrated a stimulating effect on the functional activity of macrophages and ability to produce $\mathrm{NO} / \mathrm{NO} 2$ in vitro. Lactobacilli strains increased the production of IL-12 and IFN- $\gamma$ in vitro. The AFM demonstrated different degree of the cell walls elasticity in various strains of $L A B$ and bifidobacteria. Among lactobacilli the most elastic cell wall was found in L. delbrueckii subsp. bulgaricus IMV B-7281, and among bifidobacteria - in B. animalis VKL, which induced the considerable activation of the phagocytes. Probiotic strains survival in 
the macrophages depended on the elasticity of bacterial cell walls and on the time of their joint cultivation.

Conclusions: LAB and bifidobacteria strains stimulate immunomodulatory cytokines and active oxygen and nitrogen oxides compounds production in macrophages. Strains with a more elastic cell wall according to AFM data demonstrated higher resistance to intracellular digestion in macrophages and higher level of their activation. AFM might be considered as a fast and accurate method to assess parameters of probiotic strains cell wall to predict their beneficial immunemodulatory properties. Further large-scale preclinical research needed for future application in rheumatic diseases treatment and prevention.

\section{REFERENCES:}

[1] Bubnov RV, et al. EPMA J. 2015;6:14. https://doi.org/10.1186/s13167-0150036-0

[2] Reid G, et al. Benef Microbes 2017;8(4):521-533. https://doi.org/10.3920/ BM2016.0222

[3] Bubnov RV, Babenko LP, Lazarenko LM, et al. EPMA Journal 2017;8:357. https://doi.org/10.1007/s13167-017-0117-3

[4] Mokrozub VV, et al. EPMA Journal 2015;6:13. https://doi.org/10.1186/ s13167-015-0035-1

Disclosure of Interest: None declared

DOI: 10.1136/annrheumdis-2018-eular.6888

\section{AB0044 HYPER IG-D SYNDROME TREATMENT WITH HYDROXYCHLOROQUINE}

M.P. Abi Saab ${ }^{1}$, R.M. Alsmadi ${ }^{2} .{ }^{1}$ Rheumatology; ${ }^{2}$ Dermatology, Al Ahli Hospital, Doha, Qatar

Background: Hyper IgD syndrome is a minor form of mevalonate kinase deficiency caused by a its gene mutation and considered an auto-inflammatory dis ease inherited in an autosomal recessive manner, characterised by periodic episodes of fever, arthralgia, lymphadenopathy, skin rash, headaches, and abdominal pain. These attacks can occur spontaneously or be triggered by infections. Treatment options include courses of NSAIDS, steroids, colchicine, statins I.V immunoglobulin, Cyclosporine, anti-IL-1 agents (anakinra or canakinumab) and anti-TNF antagonist Etanercept, with transient response. To date, there is no report with long term hydroxychloroquine treatment ${ }^{1-3}$.

Objectives: To report a case of Hyper lg-D syndrome with multiple recurrent episodes and successful treatment using Hydroxychloroquine alone.

Methods: We report a case of 36-year-old female, presented with a first manifestation at age 35 with fever and extended cellulitis of the left arm treated success fully with antibiotic, rapidly followed with urticarial rash involving the upper side of the body, face and upper limbs migratory type lasting less than 24 hours suspecting allergic reaction. She was under supportive treatment (anti-histaminic and low dose steroids) that improved her condition temporarily. She developed multiple recurrent similar episodes for 7 months with high grade fever up to $39^{\circ} \mathrm{C}$, inflammatory polyarthralgia affecting small joints, recurrent left elbow erythematous plaque with few subcutaneous nodules, considered as panniculitis type lesion (Picture-1) along with urticarial rash. Series of tests were undertaken along with skin biopsy.

Results: Biopsy of the lesion showed intense deep dermal neutrophil infiltrates. CRP fluctuating between 50 to $95 \mathrm{mg} / \mathrm{L}$ during the episodes. Neutropenia (1.7 to 1.9), Normal findings for ANA and SSA antibodies, ACE, Lysozyme enzyme, C3 and C4. Other infectious work-up was negative including Quantiferon God test. Protein electrophoresis found hypergammaglobulinemia. The Ig-D level was $263 \mathrm{mg} / \mathrm{L}$ and on another occasion $313 \mathrm{mg} / \mathrm{L}$ (normal <153 mg/L), the samples were taken during flare up and after recovery. The patient was treated by Colchicine for more than 3 months without beneficial effect. A trial with hydrochochloroquine $400 \mathrm{mg}$ daily brought a progressive improvement with lesser episodes at 3rd month and no recurrences at 6 months. After one year of therapy the patient was symptomless on a lower dose $200 \mathrm{mg}$ daily with completely normalised inflammatory markers.

Conclusions: We conclude that Hyper lg-D syndrome can be treated by hydroxychloroquine. This is the first report in the literature conducting this treatment option that can bring attention for further case by case trials.

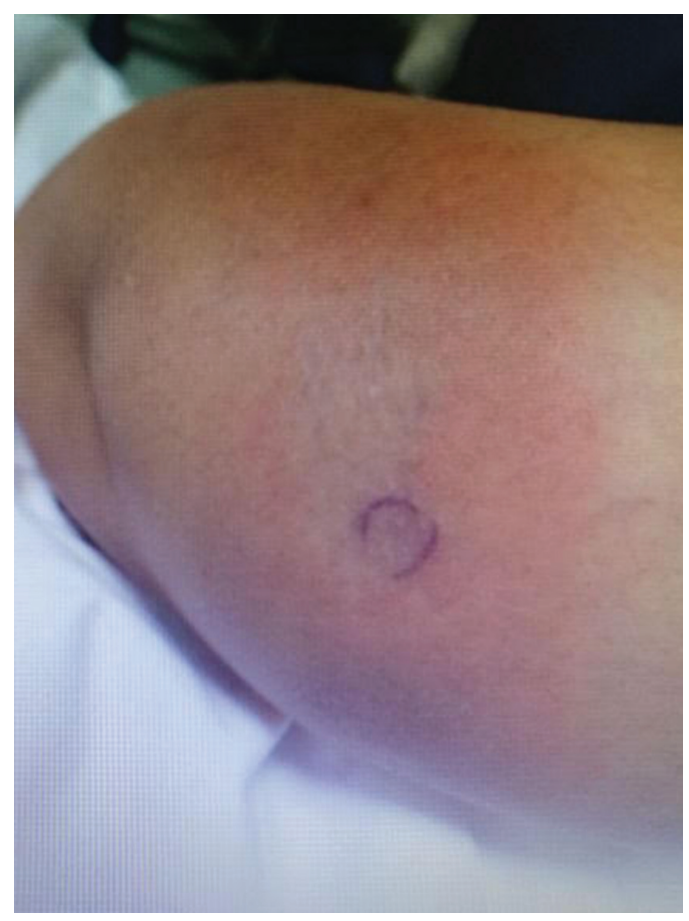

Abstract AB0044 - Figure 1

\section{REFERENCES}

[1] van der Meer JW, Vossen JM, Radl J, van Nieuwkoop JA, Meyer CJ, Lobatto S, van Furth R. Hyperimmunoglobulinaemia D and periodic fever: a new syndrome. Lancet 1984;1(8386):1087-1090.

[2] Drenth JP, Goertz J, Daha MR, van der Meer JW. Immunoglobulin D enhances the release of tumor necrosis factor-alpha, and interleukin-1 beta as well as interleukin-1 receptor antagonist from human mononuclear cells. Immunology. 1996;88(3):355-362.

[3] Bodar EJ, Kuijk LM, Drenth JP, van der Meer JW, Simon A, Frenkel J. On-demand anakinra treatment is effective in mevalonate kinase deficiency. Ann Rheum Dis. 2011;70(12):2155-2158.

Acknowledgements: Pathology department. Al Ahli Hospital, Doha, Qatar. Dermatology department. Al Ahli Hospital, Doha, Qatar

Disclosure of Interest: None declared

DOI: 10.1136/annrheumdis-2018-eular.6929

\section{AB0045 ACTIVATED RNASE L AS A NOVEL DISEASE ACTIVITY BIOMARKER IN PSORIATIC ARTHRITIS}

S.K. Zarabi ${ }^{1}$, M. Hasipek ${ }^{2}$, Y. Guan ${ }^{2}$, E. Husni ${ }^{1}$, J. Ko ${ }^{3}$, A. Kontzias ${ }^{1}$, B.K. Jha ${ }^{2}$ ${ }^{1}$ Rheumatology; ${ }^{2}$ Taussig Cancer Institute - Cancer Center Research; ${ }^{3}$ Pathology, Cleveland Clinic, Cleveland, USA

Background: Almost $60 \%$ of psoriasis ( $\mathrm{PsO}$ ) patients with psoriatic arthritis(PsA) are estimated to be untreated, undertreated or/and undiagnosed. ${ }^{1}$ Delayed diag nosis leads to permanent joint damage causing major functional decline and diminished quality of life. However, in the absence of diagnostic biomarkers, the diagnosis of psoriatic arthritis is clinical and hence difficult to establish from nonrheumatologists. ${ }^{2}$ Recent studies have demonstrated the upregulation of type interferon (IFN)-inducible genes in paired peripheral blood cells (PBC) and synovial biopsies of patient with $\mathrm{PsA}^{3}$ Oligoadenylate synthetases (OAS) are type IFN-stimulated family of proteins that are activators of the latent Ribonuclease $L$ (RNase L) pathways. The OAS-RNase L system is a potent host antiviral IFN responsive system that is completely inactive in normal conditions, but once activated mediates a broad array of pro-inflammatory cellular processes. ${ }^{4}$ 